\title{
Coverage, efficacy or dosing interval: which factor predominantly influences the impact of routine childhood vaccination for the prevention of varicella? A model-based study for Italy
}

Katsiaryna Holl ${ }^{1 * \dagger}$, Christophe Sauboin ${ }^{1 \dagger}$, Emanuele Amodio ${ }^{2,5}$, Paolo Bonanni ${ }^{3}$ and Giovanni Gabutti ${ }^{4}$

\begin{abstract}
Background: Varicella is a highly infectious disease with a significant public health and economic burden, which can be prevented with childhood routine varicella vaccination. Vaccination strategies differ by country. Some factors are known to play an important role (number of doses, coverage, dosing interval, efficacy and catch-up programmes), however, their relative impact on the reduction of varicella in the population remains unclear. This paper aims to help policy makers prioritise the critical factors to achieve the most successful vaccination programme with the available budget.
\end{abstract}

Methods: Scenarios assessed the impact of different vaccination strategies on reduction of varicella disease in the population. A dynamic transmission model was used and adapted to fit Italian demographics and population mixing patterns. Inputs included coverage, number of doses, dosing intervals, first-dose efficacy and availability of catch-up programmes, based on strategies currently used or likely to be used in different countries. The time horizon was 30 years.

Results: Both one- and two-dose routine varicella vaccination strategies prevented a comparable number of varicella cases with complications, but two-doses provided broader protection due to prevention of a higher number of milder varicella cases. A catch-up programme in susceptible adolescents aged 10-14 years old reduced varicella cases by $27-43 \%$ in older children, which are often more severe than in younger children. Coverage, for all strategies, sustained at high levels achieved the largest reduction in varicella. In general, a $20 \%$ increase in coverage resulted in a further $27-31 \%$ reduction in varicella cases. When high coverage is reached, the impact of dosing interval and first-dose vaccine efficacy had a relatively lower impact on disease prevention in the population. Compared to the long (11 years) dosing interval, the short (5 months) and medium (5 years) interval schedules reduced varicella cases by a further $5-13 \%$ and $2-5 \%$, respectively. Similarly, a $10 \%$ increase in first-dose efficacy (from 65 to $75 \%$ efficacy) prevented 2-5\% more varicella cases, suggesting it is the least influential factor when considering routine varicella vaccination.

Conclusions: Vaccination strategies can be implemented differently in each country depending on their needs, infrastructure and healthcare budget. However, ensuring high coverage remains the critical success factor for significant prevention of varicella when introducing varicella vaccination in the national immunisation programme.

Keywords: Varicella, Routine varicella vaccination impact, Coverage, Efficacy, Dosing interval

\footnotetext{
* Correspondence: Katsiaryna.x.holl@gsk.com

${ }^{\dagger}$ Equal contributors

'GSK Vaccines, Avenue Fleming 20, 1300 Wavre, Belgium

Full list of author information is available at the end of the article
} International License (http://creativecommons.org/licenses/by/4.0/), which permits unrestricted use, distribution, and reproduction in any medium, provided you give appropriate credit to the original author(s) and the source, provide a link to the Creative Commons license, and indicate if changes were made. The Creative Commons Public Domain Dedication waiver (http://creativecommons.org/publicdomain/zero/1.0/) applies to the data made available in this article, unless otherwise stated. 


\section{Background}

Varicella is a highly infectious childhood disease caused by varicella-zoster virus (VZV). Each year, the total number of varicella cases in all age groups more or less equals the size of the birth cohort, in countries with no routine varicella vaccination (RVV) programme [1]. In addition, seroprevalence studies in Europe have shown that over $90 \%$ of people have been infected with varicella by 10-15 years of age [2].

Many patients with varicella will visit a general practitioner (GP) and 2-6\% of those cases will have complications, while the annual incidence of varicella hospitalisations in Europe is 1.9 to 5.8 per 100,000 population [3]. The high infection rates and associated economic burden of varicella result in a significant public health burden [4].

Varicella infection can be prevented through effective varicella vaccines with well-established immunogenicity, reactogenicity and safety profiles [3]. In countries where RVV programmes have been implemented, a significant reduction in the incidence and burden of varicella has been observed [3]. Therefore, the World Health Organization (WHO) recommends that a RVV programme should be considered in countries where varicella has an important public health burden and where high (>80\%) coverage can be sustained [5].

With increasing constraints on healthcare budgets, policy makers need more clarity on which vaccination strategy to choose for optimal reduction of the disease burden. Several practical aspects that need consideration include how to reach and sustain high coverage levels in order to optimise protection against varicella disease. Other factors that influence the impact of vaccination include the choice of using either one-dose or two-dose vaccination schedules, either short (months) or long (years) intervals between two doses, and whether or not a catchup programme should be implemented when introducing childhood RVV. RVV will be introduced in Italy as a national programme in the near future, following analysis of the vaccine data from eight pilot regions which have already implemented it. An Interregional Group on Varicella Vaccination (IGVV) was established in 2013 to assess the varicella vaccination effectiveness using common standardised methods. Since 2003, eight Italian regions (Basilicata, Calabria, Friuli - Venezia Giulia, Apulia, Sardinia, Sicily, Tuscany and Veneto) started to introduce childhood RVV (in children aged 13-15 months and 5-6 years) in their regional immunisation programmes, using different schedules. Currently all regions use a two-dose schedule [6].

The objective of this model-based study is to help policy makers choose an appropriate vaccination strategy in their country, by exploring the relative impact of several key factors on varicella burden: (1) coverage, (2) onedose or two-dose schedule, (3) vaccine efficacy of the first dose, (4) short or long interval between doses, and (5) whether or not to implement a catch-up programme. Although this study aims to inform RVV policy makers in general, the example of Italy was used, given the diverse regional strategies currently in place and anticipated policy changes.

\section{Methods}

An age-structured dynamic transmission model was developed, based on the framework described by Brisson et al. $[7,8]$ and following the same methodology used for the adaptation for France [9], to assess the impact of varicella vaccination on disease burden by varying the key influential vaccination strategy parameters. The model predicts both varicella and zoster diseases over a lifetime with and without vaccination to reflect the full picture of the disease burden related to VZV. The model structure shows varicella disease states as susceptible, latent or exposed, infectious, and recovered. Factors such as waning of vaccine efficacy over time and the influence of population migration on infection rates were included. The model was adapted to match the epidemiology of varicella prior to vaccination in Italy using an empirically-derived contact matrix [10]. A detailed description of the model can be found in Additional file 1. The incidence of varicella before vaccination was compared to the predicted incidence over time following the introduction of vaccination in the model. Based on these comparisons, the percentage reduction in varicella cases is presented postvaccine introduction for all combinations of dosing, coverage and efficacy tested. After introducing childhood RVV, milder 'breakthrough' cases of varicella can occur in the model. The predicted incidence of varicella over time for each scenario is presented below.

\section{Model assumptions and scenarios (see Table 1)}

The model assumed that the maximum vaccine coverage would gradually be attained in the first 3 years of introducing the RVV programme. Three vaccination coverage scenarios were considered for dose one \& dose two; including high (i.e., 95 \& $80 \%$ ), medium (i.e., 85 \& $70 \%$ ) and low coverage (i.e., 75 \& $60 \%$ ).

Two different values were assessed for vaccine efficacy of the first dose (i.e., 65 or $75 \%$ ). For two-dose schedules, vaccine efficacy following the second dose was fixed at $95 \%$ [11].

Three scenarios were considered to assess the impact of a longer or shorter interval between the two vaccine doses; including a 5-month, a 5-year and an 11-year interval. These intervals were based on current Measles, Mumps and Rubella (MMR) schedules used in different countries, while in Italy several regions have typically used a 4-or 5-year interval between doses. 
Table 1 Scenario analyses

\begin{tabular}{|c|c|c|}
\hline Parameter & Values & Source \\
\hline $\begin{array}{l}\text { Vaccination coverage: } \\
1^{\text {st }} \text { dose } \& 2^{\text {nd }} \text { dose (VC D1_D2) }\end{array}$ & $\begin{array}{l}95-80 \% \\
85-70 \% \\
75-60 \%\end{array}$ & Assumptions \\
\hline $\begin{array}{l}\text { Vaccine efficacy (VE): } \\
\text { for } 1^{\text {st }} \text { dose }\end{array}$ & $\begin{array}{l}65 \% \\
75 \%\end{array}$ & $\begin{array}{l}\text { Prymula et al. [11] } \\
\text { Assumption }\end{array}$ \\
\hline Vaccine efficacy (VE) for $2^{\text {nd }}$ dose & $95 \%$ (fixed) & Prymula et al. [11] \\
\hline $\begin{array}{l}\text { Dosing interval scenarios } \\
\text { Age at } 1^{\text {st }} \text { dose } \& 2^{\text {nd }} \text { dose }\end{array}$ & $\begin{array}{l}\text { Short ( } 1-1.5 \text { years): } 13 \text { months \& } 1.5 \text { years } \\
\text { Medium ( } 1-6 \text { years): } 13 \text { months \& } 6 \text { years } \\
\text { Long ( } 1-12 \text { years): } 13 \text { months \& } 12 \text { years }\end{array}$ & Assumptions \\
\hline Catch-up programme & For susceptible 12-year-olds, for 11 years, $20 \%$ coverage & Assumptions \\
\hline
\end{tabular}

All dosing scenarios included a catch-up programme in adolescents (12-year-olds) who were still susceptible to varicella (i.e., not vaccinated and had not yet had varicella). The catch-up programme, with a coverage of $20 \%$, was modelled for the first 11 years since introducing vaccination. From the twelfth year, the original vaccinated cohort will have reached the age of 12 years, and would therefore be protected. A scenario presents the impact of not including a catch-up programme.

\section{Results}

Increasing coverage or efficacy, or shortening the dosing interval had a positive effect on the prevention of varicella but each to a different extent. The impact of various vaccination strategies on varicella prevention are presented below.

\section{Routine varicella vaccination of toddlers and children: impact of the number of doses}

The first practical issue to consider when implementing RVV is the choice to use a one- or two-dose vaccination programme. As per our modelling results, a one-dose schedule effectively controls varicella disease albeit with the presence of mild breakthrough cases, whilst a twodose schedule provides optimal protection against mild and severe varicella with the potential to reduce breakthrough varicella cases in the population. The baseline varicella incidence before introduction of RVV was 910.4 per 100,000 population per year. Varicella incidence decreased immediately after introduction of the vaccine, with by year 30 around $70 \%$ reduction (incidence of 273.0/100,000) and around $40 \%$ reduction $(546.0 / 100,000)$ across all age groups for two- and onedose strategies, respectively (Fig. 1). The overall number of complications due to varicella was 43 per 100,000 population per year. The reduction in number of complications in the first 15 years of post-introduction of vaccination was substantial (up to $90.1 \%$ for two-dose and up to $84.6 \%$ for one-dose strategies). Between 15 and 30 years post - introduction of vaccination, there was a peak in complications with one-dose strategies (up to $18.7 / 100,000$ after 19 years), reflecting the peaks in incidence (Fig. 3), and due to accumulation of nonvaccinated varicella cases. However, this difference became minimal $(6.0 \%)$ between the two strategies after 30 years of vaccination (Fig. 2)

\section{Routine varicella vaccination of toddlers and children: impact of the coverage}

Whether considering a one-dose or two-dose vaccination strategy, it is important to understand the impact of vaccination coverage on varicella disease burden in the population. Within each vaccination strategy considered in the model, it is apparent that high coverage was the key driver of vaccine impact on varicella (Fig. 1).

A low coverage reduced varicella by $64.1,58.3$ and $55.0 \%$ (to 327,380 and $410 / 100,000$ ) with a two-dose strategy and short, medium and long dosing intervals, respectively. When coverage was high, an additional 27.2$31.1 \%$ reduction in varicella cases was observed. The disproportionate improvement in outcomes (i.e., vaccinating $20 \%$ more children provides around $30 \%$ fewer varicella cases in the population) highlights the additional indirect benefit vaccination provides at higher coverage levels, by providing herd immunity (Figs. 1 and 3 ).

The effect of a $10 \%$ increase from low to medium coverage and from medium to high coverage, respectively, was to reduce varicella cases by an additional $15.0 \%$ (to $190.1 / 100,000)$ and $12.2 \%(78.8 / 100,000)$ with the short dosing interval, $12.7 \%(263.7 / 100,000)$ and $17.5 \%(104.1 / 100,000)$ with the medium dosing interval, and, $11.1 \%(309.1 / 100,000)$ and $19.9 \%(127.4 / 100,000)$ with the long dosing interval (see Fig. 1).

\section{Routine varicella vaccination of toddlers and children: impact of the dosing interval}

Within two-dose schedules, there is further debate about whether a short-term (5 months) or longer term (5 or 11 years) interval should be used.

Shortening the dosing interval resulted in an additional 3-8 \% reduction in varicella cases (from 5 years dosing interval to 6 months) and 2-5\% reduction (from 
a Percent reduction in incidence per scenario
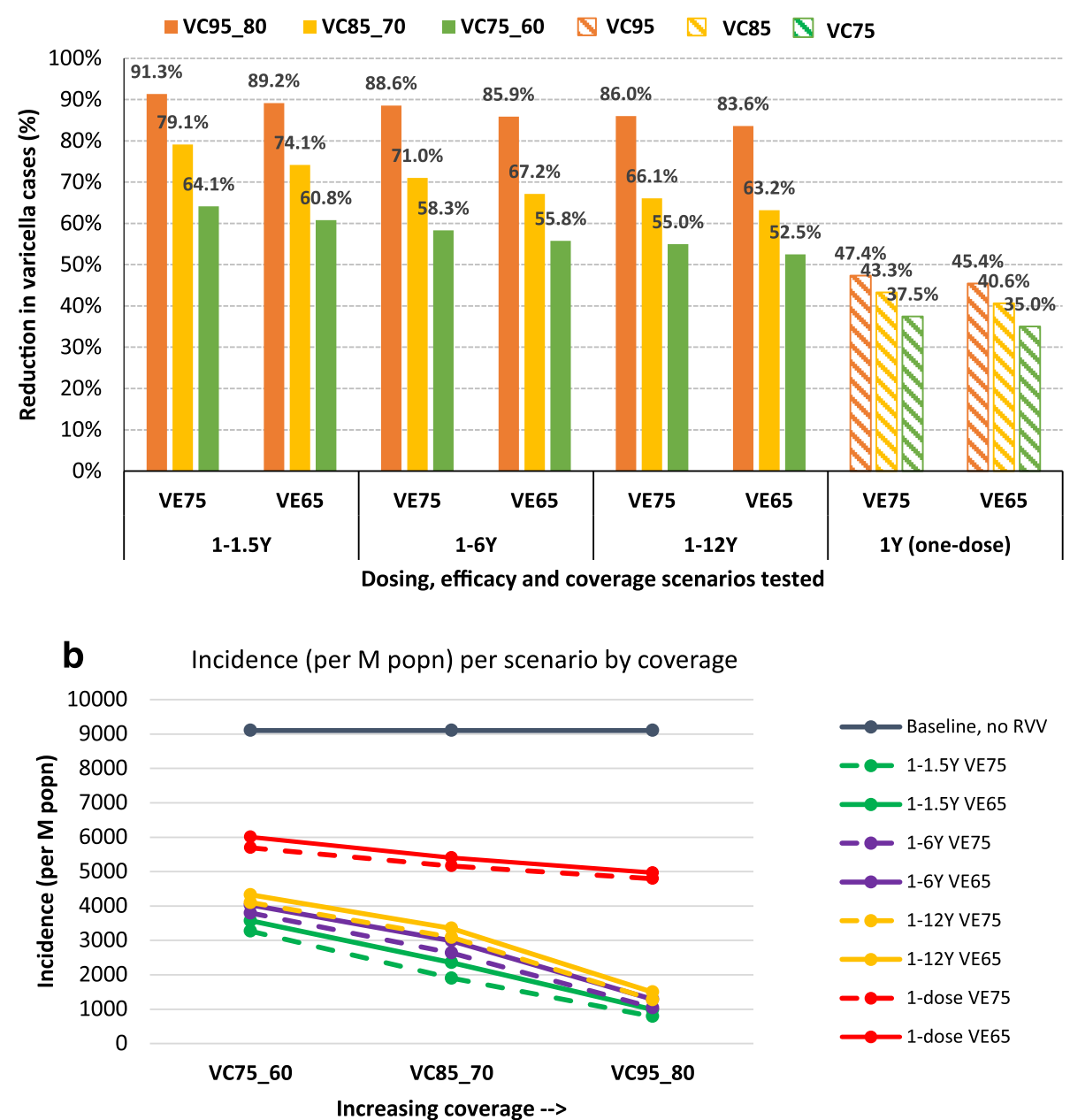

Fig. 1 Percentage reduction in varicella incidence (a) and incidence per 100,000 popn (b) across all age groups at 30 years after vaccination, for different coverage, efficacy and dosing scenarios compared with no varicella vaccination. Legend: VC (vaccine coverage $1^{\text {st }}$ dose_ $2^{\text {nd }}$ dose), VE ( $1^{\text {st }}$ dose vaccine efficacy)

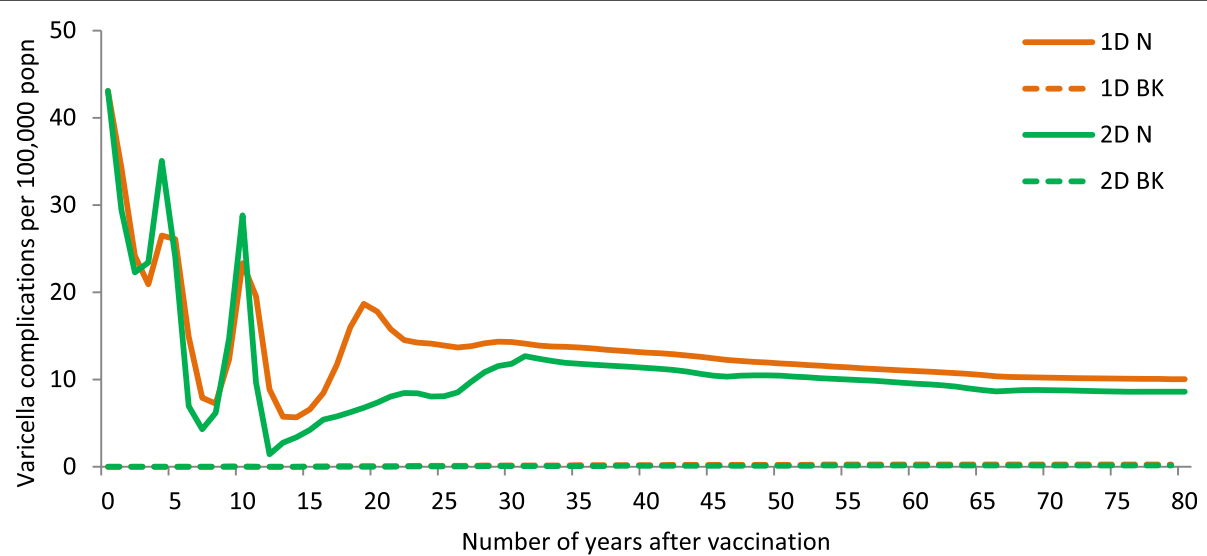

Fig. 2 Complications among natural (N) and breakthrough (BK) varicella cases with a one-dose and two-dose vaccination strategy. Legend: 1D (one-dose), 2D (two-dose), N (natural), BK (breakthrough). Note: Complications among breakthrough cases were close to 0 with both one-and two-dose strategies 

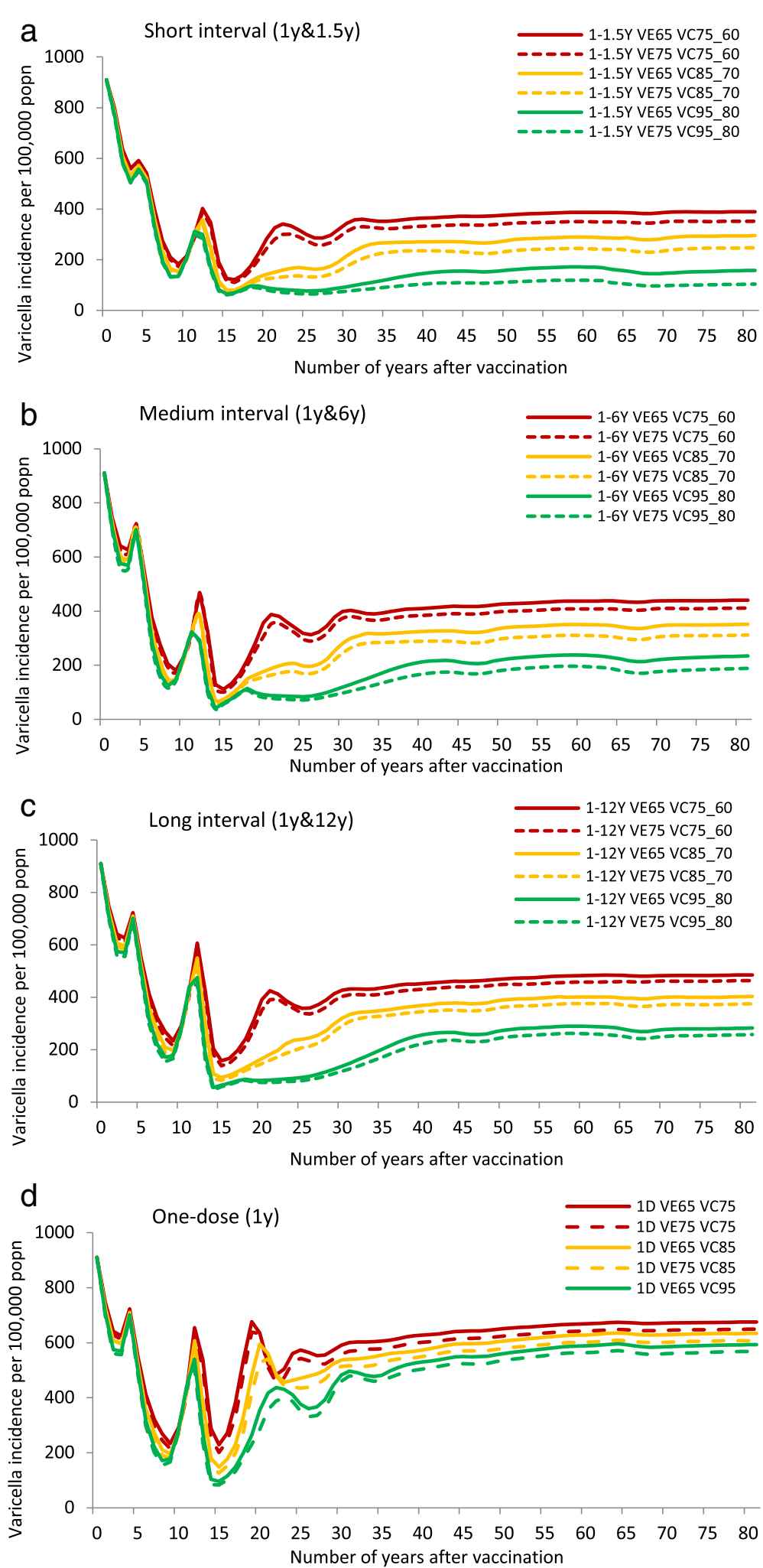

Fig. 3 Varicella incidence (per 100,000 population) following RW for each scenario. Legend: VE (vaccine efficacy), VC (vaccine coverage $1^{\text {st }}$ dose_ $2^{\text {nd }}$ dose), $1 \mathrm{D}$ (one-dose) 
11 years dosing interval to 5 years). The overall 5-13\% reduction in number of varicella cases was observed when 6 months between the two doses was considered instead of 11 years (Fig. 1). Changes in dosing interval appear to have a smaller impact on varicella burden than changes in coverage.

\section{Routine varicella vaccination of toddlers and children: impact of the first-dose efficacy}

With a one-dose vaccination schedule and $75 \%$ vaccine efficacy post-dose one, a $37.5 \%$ (to $569 / 100,000$ ) and $47.4 \%(479 / 100,000)$ reduction in varicella cases was observed with low and high coverage, respectively. With $65 \%$ efficacy post-dose one, the percentage of varicella cases prevented was $35.0 \%(601 / 100,000)$ and $45.4 \%(497 / 100,000)$, which is only slightly lower (2-3 \%), showing that a $10 \%$ drop in efficacy of dose one would not have a significant impact on overall disease prevention.

Similarly with a two-dose vaccination schedule and $75 \%$ efficacy post-dose one, the reduction in varicella cases ranged from $55.0 \%$ (low coverage and long interval) to $91.3 \%$ (high coverage and short interval). This was comparable to the $52.5 \%$ (low coverage and long interval) to $89.2 \%$ (high coverage and short interval) reduction observed with $65 \%$ efficacy post-dose one (Fig. 1).

\section{Routine varicella vaccination of toddlers and children: impact of a catch-up programme}

The impact of a catch-up programme on disease burden was assessed for both a one-dose and two-dose strategy, using a coverage of $85 \%$ for the first dose and $70 \%$ for the second dose. Figure 4 shows that the catch-up programme substantially reduced the second peak in incidence of varicella that is predicted to occur around ten years after introducing childhood RVV, by $47.4 \%$ (to $350.8 / 100,000)$ with a two-dose strategy and by $25.5 \%$ (to $428.4 / 100,000$ ) with a one-dose strategy. Without introduction of catch - up programme the incidence of varicella at around 10 years post - introduction of vaccination was 667.6 and 575.2/100,000 for a two-dose and one-dose strategy, respectively (Fig. 4a and b).

Figure 5 shows the breakdown of varicella cases by age group predicted to occur at given time points after introducing childhood RVV, with and without a catchup programme, using the example of the two-dose strategy. The impact of the catch-up programme ten years after introducing childhood RVV was to significantly reduce the overall incidence of varicella (by $47.4 \%$, from 667.6 to $350.8 / 100,000$ ), as well as to reduce the incidence in the 10-14 year age group (by $79.9 \%$, from 94.8 to $19.1 / 100,000$ ) compared with having no catch-up programme.

\section{Discussion}

The WHO position is that countries should consider introducing varicella vaccination into the routine immunisation programme where varicella is an important public health burden. They recommend that these countries have sufficient resources to maintain high coverage. Currently, eight countries recommend childhood RVV in the European Union, either at the national or regional level, while sixteen countries recommend targeted vaccination of susceptible teenagers or at-risk groups [3]. Several practical factors related to vaccination strategy need to be taken into consideration as they play a key role in determining the impact of a RVV programme on varicella cases in the population; such as how to optimise coverage, choice of dosing strategy and whether to implement a catch-up programme.

Following the introduction of childhood RVV, there is a period when incidence rates fluctuate, this is common with infectious disease models which typically assume homogenous contacts and transmission risk within each age group and include a dynamic relation between the force of infection, the proportion of infectious and susceptible individuals. In our case, other factors can influence these variations such as the highly infectious nature of varicella, the changes in coverage rates over time and the temporary implementation of a catch-up programme.

This study considered many possible vaccination scenarios such as one- or two-dose schedule, different coverage rates, dosing intervals, efficacy post-dose one and availability of a catch-up programme to predict the reduction of varicella burden in the population following a childhood RVV programme. The results of this analysis have shown that the most influential factor in reducing varicella incidence was high coverage. A shorter interval between two doses improved outcomes further, while the impact of a $10 \%$ change in first-dose efficacy was less significant whether with a one- or two-dose strategy.

Varicella vaccination programmes have been implemented in different ways, with some countries implementing a childhood RVV programme while other countries target only high-risk groups. Among countries with a RVV programme, a one-dose schedule has been publicly funded in Australia since 2005 [12] with a coverage of over $80 \%$ resulting in important reductions in varicella morbidity and mortality $[13,14]$. Whereas in the USA and Germany, the initial one-dose schedule was replaced by a two-dose schedule after some years, resulting in even greater disease prevention in the population $[15,16]$. Breakthrough varicella is thought to be caused mainly by primary vaccine failure rather than vaccine efficacy waning [17]. This could explain why countries implementing a two-dose schedule were able 

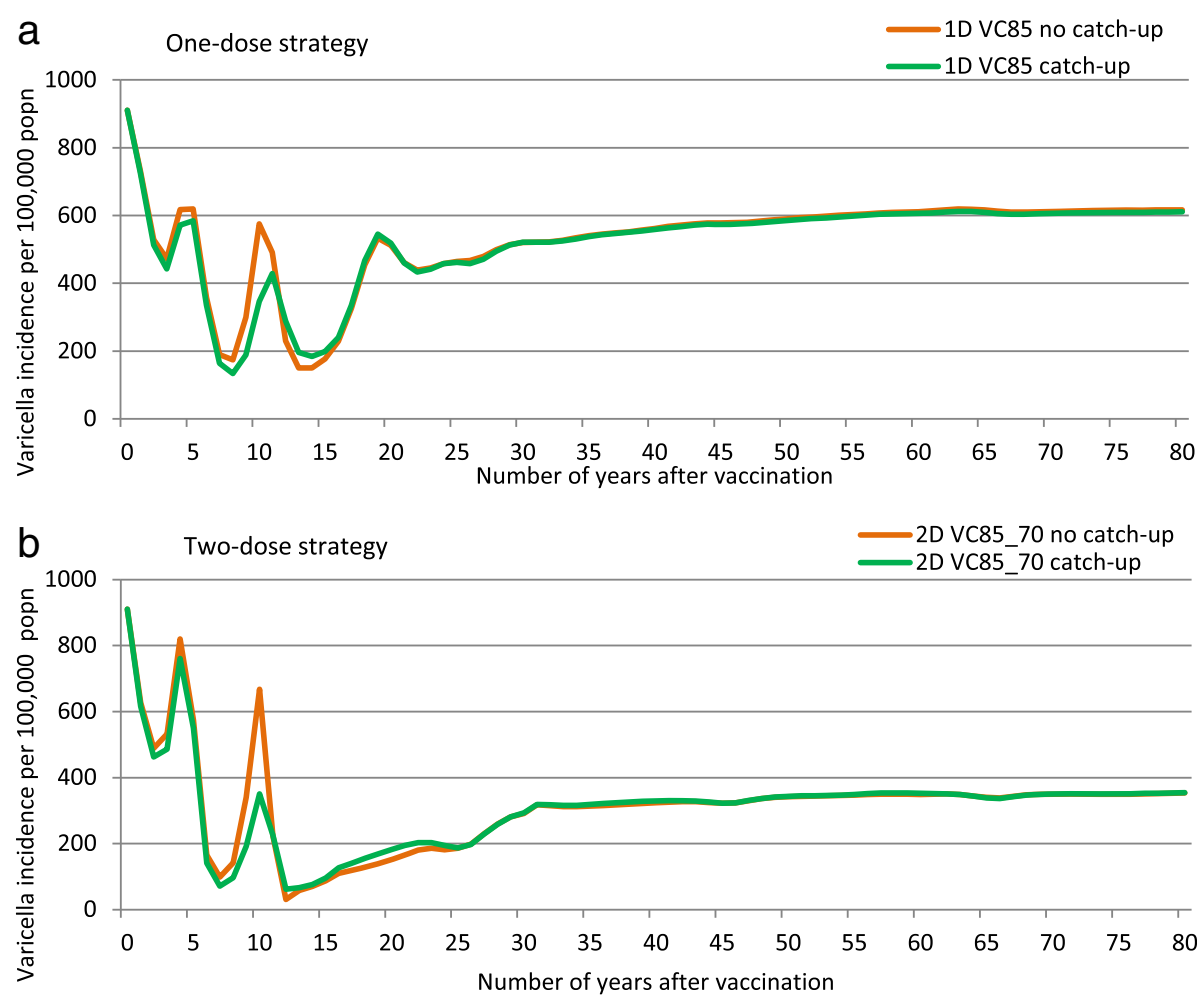

Fig. 4 Influence of a catch-up programme on varicella incidence with a one-dose and two-dose strategy. Legend: 1D (one-dose), 2D (two-dose), VC (vaccine coverage $1^{\text {st }}$ dose $\_2^{\text {nd }}$ dose)

to further reduce the incidence of breakthrough cases compared to those using one-dose schedules [3]. Data from active surveillance in the USA show that a onedose schedule was able to significantly decrease the number, size and duration of varicella outbreaks, however the addition of a second dose was able to nearly eliminate outbreaks [3]. When comparing a one-dose and two-dose strategy in this study, both were found to significantly reduce the burden of varicella, however the impact with a two-dose strategy was greater. Although a one-dose vaccination strategy prevented fewer varicella cases, its prevention of severe cases (i.e., with complications) was comparable to two-dose strategies. Evidence from countries with childhood RVV supports the finding that one-dose strategies have higher efficacy against more severe than less severe varicella, while two-dose

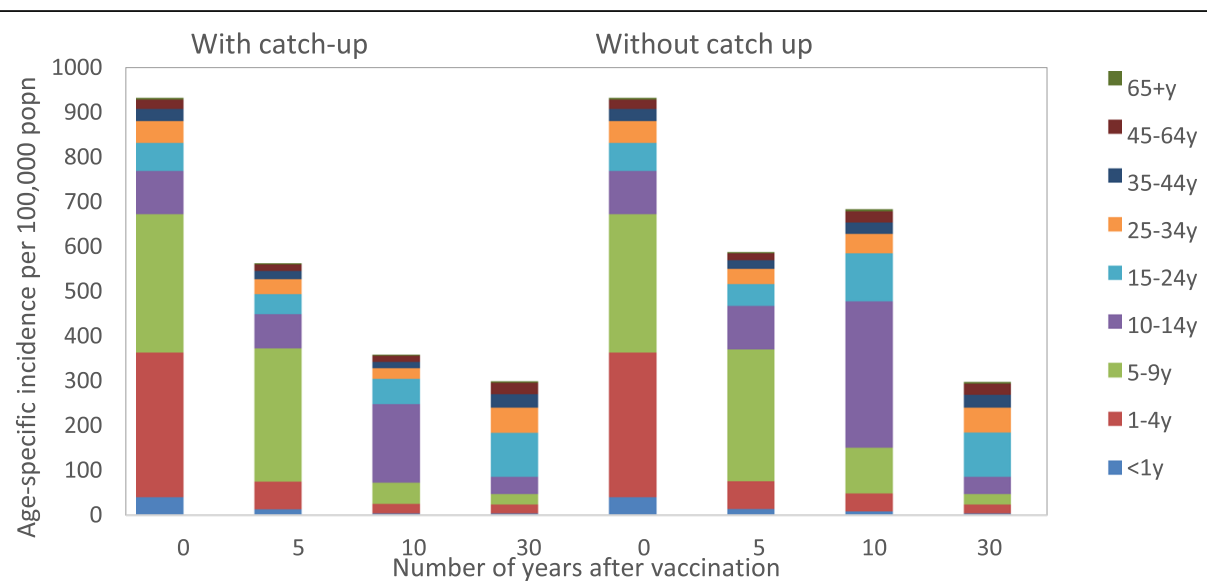

Fig. 5 Influence of a catch-up programme on age-specific incidence of varicella (two-dose scenario). Legend: $y$ (year). Note: The vaccine strategy in Fig. 5 included dosing at $1 y$ and $6 y$ with coverage of 85 and $75 \%$ respectively 
strategies have high efficacy against any varicella regardless of severity [3]. Therefore, the main benefit of a onedose schedule appears to be in reducing mortality and severe morbidity, while a two-dose schedule has been found to reduce the disease burden irrespective of severity and to prevent cases of breakthrough infection [18].

Maintaining high vaccine coverage is critical to providing substantial protection for the population, whether with a one-dose or two-dose vaccination strategy, given the highly infectious nature of VZV. The model findings are consistent with WHO recommendations in that sustaining coverage of $85 \%$ or higher with a RVV programme will have an optimal impact. Our findings show that a high coverage was also able to compensate for other vaccination strategies which may be less effective at reducing varicella burden, such as longer interval between doses and lower vaccine efficacy post-dose one. In countries where childhood RVV has been implemented with high coverage rates, surveillance data have shown significant and rapid reductions in varicella disease burden (cases, complications, hospitalisations and deaths) observed in all age groups including nonvaccinated age groups such as infants and adults, demonstrating herd protection effects [3].

This study found that shortening the dosing interval increased the number of cases prevented, although the effect was less important than increasing coverage rate. Another benefit of a short interval between doses is that this could help reduce the risk of breakthrough varicella in the interval between doses [17]. In Italian regions where childhood RVV has already been introduced, the first dose is given around the $13^{\text {th }}$ to $15^{\text {th }}$ month of life and the second dose around the $5^{\text {th }}$ to $6^{\text {th }}$ year of life; thus with a 4- to 5-year interval between doses [6]. There is evidence from three recent Italian studies showing that a long-interval schedule does not significantly affect the performance of a two-dose varicella vaccination programme $[6,19,20]$. These studies reported very high rates of disease reduction across eight regions of Italy (Apulia, Basilicata, Calabria, Friuli-Venezia Giulia, Sardinia, Sicily, Tuscany and Veneto) that implemented childhood RVV between 2003 and 2013; overall incidence decreased from 6.7 per 1000 population in 2003 to 1.7 per 1000 population in 2012. In Sicily, which was the first Italian region to implement varicella vaccination, there was a $95 \%$ reduction in cases over 10 years, with coverage rates increasing to $85 \%$ [20]. So while a shorter interval favours fewer cases of varicella, the largest driver of prevention is higher coverage. In the USA, despite a long interval between doses [21], a clear benefit of vaccination was observed with a high coverage of $90 \%$ for infants targeted for vaccination [22, 23]. This supports the finding that coverage is a more important factor than dosing interval in determining the success of a childhood RVV programme. When deciding on dosing schedules, countries should therefore consider fitting in varicella vaccinations with their current immunisation schedule, by assessing the number of vaccines currently given at different ages and the timing of visits, to help improve coverage for their RVV programme. Combining the second dose of varicella vaccination with a scheduled visit for other childhood vaccinations could help to increase coverage of the second dose.

The choice of using MMR with a single antigen varicella vaccine $(M M R+V)$ or the combined MMRV depends on country-specific preferences, given the uncertainty surrounding potential increased febrile convulsion risks with the first dose of MMRV. An association was seen in clinical trials and observational studies between the administration of the first dose of MMRV and febrile convulsions, compared with MMR + $\mathrm{V}$ vaccination $[24,25]$. German data found one additional case of febrile convulsion with MMRV per 5882 or 2747 vaccinees compared with MMR or MMR + V, respectively $[24,26]$. Other studies also found a small increased risk of febrile convulsions in the second week post-vaccination [26-28]. By contrast, the region of Tuscany in Italy decided to keep MMRV for the first dose as they had not observed an increased risk of any adverse events due to MMRV compared with MMR + V [29]. In another study, the incidence febrile convulsions in the 40-day post-vaccination period did not differ between MMR $+\mathrm{V}$ and MMRV. There was a minimal impact of MMRV on the overall population risk of febrile convulsions, with an excess risk of 3.52 febrile convulsions per 10,000 vaccinees compared to MMR + V [27]. There was no change in the baseline incidence of febrile convulsions in the population as a whole following the introduction of MMRV. The small increased risk of febrile convulsions occurring within the first 2 weeks of administration of MMRV vaccine must be balanced against administering separate MMR and varicella vaccines $[26,28]$, potentially impacting vaccine uptake [30]. The eight Italian regions that provided RVV offered two doses of MMRV, MMR + V or single antigen $V$, given at different times and sometimes in combination with appointments for different vaccines [6]. Coverage is linked to dosing strategy; in countries where there is a high MMR coverage, switching to MMRV does not appear to affect coverage, but switching to $\mathrm{MMR}+\mathrm{V}$ tends to result in a lower $\mathrm{V}$ coverage. In Germany, the uptake of varicella vaccination (but not MMR) decreased by 4$19 \%$ when the national recommendations changed from the administration of MMRV to MMR + V [30]. Benefit/ risk assessment suggests that the use of MMRV instead of MMR + V can prevent an additional 1976 varicellarelated hospitalisation days per year at the cost of an additional 225 vaccine-related febrile convulsions 
hospitalisation days when coverage drops by $12 \%$. That is the trade-off between the two vaccination schemes that needs to be considered when making decisions on their use in immunisation programmes [31].

The primary goal of a varicella vaccination programme is to generate a public health impact by reducing the number of varicella cases and related hospitalisations and deaths. In 2000, using mathematical models, Brisson et al. [7] showed a temporal immediate increase in herpes zoster (HZ) incidence post-vaccination. However, medium-term epidemiological evidence is now available from several countries that have implemented varicella vaccination. The majority of these studies concluded that $\mathrm{HZ}$ incidence was either not increasing, or the increase was not directly related to varicella vaccination $[13,32$, $33]$, or that the study was not designed to prove causality between $\mathrm{HZ}$ and varicella vaccination [34, 35]. Factors that may have influenced an increase in $\mathrm{HZ}$ incidence include increasing elderly population [36], increased oral corticosteroid use [37], and, chronic comorbid conditions [38, 39]. Therefore this paper focuses solely on the impact of influential factors on varicella.

Limitations of this study relate to the underlying model assumptions for which a lack of evidence exists; such as duration of immunity after two doses or waning of natural immunity and two-dose vaccine efficacy for full or partial or non-responders. In order to focus on the influential factors of interest for this study, the same simplified catch-up programme was implemented in all dosing scenarios. Although a conservative catch-up coverage of $20 \%$ was used, the catch-up duration was 11 years based on the needs for the long-interval dosing schedule (i.e., after 11 years the first infants vaccinated would receive their second routine dose), and may therefore have artificially improved the outcomes in this dosing scenario. Despite this, the long-interval dosing scenarios had the worst predicted outcomes (among two-dose schedules). Therefore, the effect of using a simplified catch-up programme on results was expected to be minor in long-interval dosing scenarios. In this study, despite the low coverage of the catch-up programme, it was found to be beneficial in reducing the peak of cases predicted to occur around ten years after implementing RVV among older children in both one-dose and two-dose strategies. This finding has also been observed in other modelling studies of RVV [3]. All Italian regions may introduce a catch-up programme for susceptible adolescents, since this was offered by the National Vaccination Plan in 2012-2014, although coverage of the catch-up programme may vary across the different regions $[6,20]$.

The impact of different vaccination scenarios on payers' budget and cost-effectiveness ratios have not been assessed in the current study. Scenarios with higher coverage, more doses and catch - up will have increased vaccination programme cost, however, this increase needs to be balanced against expected larger cost savings due to better disease prevention (e.g., reductions in resource utilisation and lost productivity). Published economic evaluations have found that introducing both one- and two-dose RVV strategies are cost-effective from payer perspectives and even cost-saving from societal perspectives [40].

\section{Conclusion}

The current study may provide insights for policy makers on how to optimally reduce the burden of varicella in their country. Coverage of varicella vaccination is a critical factor in significantly reducing the number of varicella cases, whether for a one-dose strategy or a two-dose strategy. The combination of high coverage with dosing intervals of five years or less was more effective at preventing varicella than longer dosing intervals. A high coverage can compensate to some extent for decreases in cases prevented due to long intervals between doses. Similarly, at a lower coverage, varicella prevention was improved with short interval dosing schedules. Coverage had a greater impact on vaccination outcomes than the efficacy of the first dose or the dosing interval. The catch-up programme substantially reduced the peak in cases after ten years of introducing childhood RVV, even with a modest vaccine coverage in adolescents.

\section{Additional file}

Additional file 1: Technical appendix - dynamic transmission model for Italy. (DOCX 299 kb)

\section{Abbreviations \\ 1D: One-dose; 2D: Two-dose; BK: Breakthrough cases; GP: General practitioner; HZ: Herpes zoster; MMR: Measles, mumps and rubella; N: Natural cases; RW: Routine varicella vaccination; USA: United States of America; VC: Vaccine coverage of dose 1_dose 2; VE: Vaccine efficacy; VZV: Varicella-zoster virus; WHO: World Health Organization; y: Year

\begin{abstract}
Acknowledgements
The authors thank Federico Marchetti and Rosaria Silvestri (both GSK, Italy) and Stephane Lorenc (freelance consultant on behalf of GSK Vaccines) for their involvement and help in the analysis. They also thank Stephanie Garcia (Business \& Decision Life Sciences on behalf of GSK Vaccines) for editorial support and manuscript coordination and Kavi Littlewood (Littlewood Writing Solutions on behalf of GSK Vaccines) for medical writing support.
\end{abstract}

\section{Funding}

GlaxoSmithKline Biologicals SA funded this study (GSK study identifier: HO-14-15821) and all costs related to the development of the publication.

Availability of data and materials

All data are either detailed in the methods or supplementary material or can be found in the referenced literature. 


\section{Authors' contributions}

$\mathrm{KH}, \mathrm{CS}$ and PB conceived and designed the study, developed and populated the model, all authors analysed the data and provided substantial scientific input to the analysis. All authors had full access to the data and commented on drafts. They also all contributed to the final version and gave final approval before submission.

\section{Competing interests}

SC and HK are employees of the GSK group of companies and report ownership of stock options/restricted shares from this company. BP has received fees from the GSK group of companies and Sanofi Pasteur MSD outside the submitted work. GG acted as investigator in clinical trials for the GSK group of companies, Sanofi Pasteur MSD and Novartis, has received fees from the GSK group of companies and Sanofi Pasteur MSD for board membership, consulting fees from the GSK group of companies as well as payment for lectures from the GSK group of companies, Sanofi Pasteur MSD, Novartis and Crucell/Janssen and payment for manuscript preparation from Novartis. AE has nothing to disclose.

\section{Consent for publication}

\section{Not applicable.}

\section{Ethics approval and consent to participate} Not applicable.

\section{Author details}

${ }^{1}$ GSK Vaccines, Avenue Fleming 20, 1300 Wavre, Belgium. ${ }^{2}$ University of Palermo, Via Del Vespro 133, 90127 Palermo, Italy. ${ }^{3}$ University of Florence, Viale GB Morgagni, 48, 50134 Florence, Italy. ${ }^{4}$ University of Ferrara, Via Fossato di Mortara 64b, 44121 Ferrara, Italy. ${ }^{5}$ Present Address: Unit of Epidemiology, ATS Brianza, Viale Elvezia 2, 20900 Monza, Italy.

Received: 25 February 2016 Accepted: 30 September 2016 Published online: 21 October 2016

\section{References}

1. CDC VPD Surveillance Manual, 5th Edition, 2011 Varicella: Chapter 17-1; http://www.cdc.gov/vaccines/pubs/surv-manual/chpt17-varicella.pdf Accessed 6 June 2016

2. Nardone A, de Ory F, Carton M, Cohen D, van Damme P, Davidkin I, et al. The comparative sero-epidemiology of varicella zoster virus in 11 countries in the European region. Vaccine. 2007;25(45):7866-72.

3. European Center for Disease Prevention and Control Guidance. Varicella vaccination in the European Union. ECDC; 2015. http://ecdc.europa.eu/en/ publications/Publications/Varicella-Guidance-2015.pdf. Accessed 1 July 2016.

4. Trucchi C, Gabutti G, Rota MC, Bella A. Burden of varicella in Italy, 2001-2010: analysis of data from multiple sources and assessment of universal vaccination impact in three pilot regions. J Med Microbiol. 2015;64(11):1387-94.

5. The World Health Organization. Varicella and herpes zoster vaccines: WHO position paper Wkly Epidemiol Rec. 2014;89(25):265-88.

6. Bechini A, Boccalini S, Baldo V, Cocchio S, Castiglia P, Gallo T, et al. Impact of universal vaccination against varicella in Italy: experiences from eight Italian regions. Hum Vaccin Immunother. 2015;11(1):63-71.

7. Brisson M, Edmunds WJ, Gay NJ, Law B, De Serres G. Modelling the impact of immunization on the epidemiology of varicella zoster virus. Epidemiol Infect. 2000;125:651-69.

8. Brisson M, Melkonyan G, Drolet M, De Serres G, Thibeault R, De Wals P. Modeling the impact of one- and two-dose varicella vaccination on the epidemiology of varicella and zoster. Vaccine. 2010;28(19):3385-97.

9. Ouwens MJ, Littlewood KJ, Sauboin C, Téhard B, Denis F, Boëlle PY, et al. The impact of 2-dose routine measles, mumps, rubella, and varicella vaccination in France on the epidemiology of varicella and zoster using a dynamic model with an empirical contact matrix. Clin Ther. 2015;37(4):816-29.

10. Mossong J, Hens N, Jit M, Beutels P, Auranen K, Mikolajczyk R, et al. Social contacts and mixing patterns relevant to the spread of infectious diseases. PLoS Med. 2008;5(3):e74.

11. Prymula R, Bergsaker MR, Esposito S, Gothefors L, Man S, Snegova N, et al. Protection against varicella with two doses of combined measles-mumpsrubella-varicella vaccine versus one dose of monovalent varicella vaccine: a multicentre, observer-blind, randomised, controlled trial. Lancet. 2014;383:1313-24.
12. National Centre for Immunisation Research \& Surveillance (NCIRS). Varicella history Australia (Dec 2013). http://www.ncirs.edu.au/assets/provider_resources/ historyNaricella-history-December-2013.pdf Accessed 9 October 2015.

13. Heywood AE, Wang H, Macartney KK, McIntyre P. Varicella and herpes zoster hospitalizations before and after implementation of one-dose varicella vaccination in Australia: an ecological study. Bull World Health Organ. 2014;92(8):593-604.

14. Carville KS, Riddell MA, Kelly HA. A decline in varicella but an uncertain impact on zoster following varicella vaccination in Victoria, Australia. Vaccine. 2010;28(13):2532-8.

15. Streng A, Grote V, Carr D, Hagemann C, Liese JG. Varicella routine vaccination and the effects on varicella epidemiology - results from the Bavarian Varicella Surveillance Project (BaVariPro), 2006-2011. BMC Infect Dis. 2013;13:303

16. García Cenoz M. Varicella vaccination programs do not seem to shift the age of disease to older age groups. (Editorial) Transl Pediatr. 2014;3(4):275-7.

17. Bonanni P, Gershon A, Gershon M, Kulcsár A, Papaevangelou V, Rentier B, et al. Primary versus secondary failure after varicella vaccination: implications for interval between 2 doses. Pediatr Infect Dis J. 2013;32(7):e305-13.

18. Zhou F, Ortega-Sanchez IR, Guris D, Shefer A, Lieu T, Seward JF. An economic analysis of the universal varicella vaccination program in the United States. J Infect Dis. 2008;197 Suppl 2:S156-64.

19. Tafuri S, Fortunato F, Cappelli MG, Cozza V, Bechini A, Bonanni P, et al. Effectiveness of vaccination against varicella in children under 5 years in Puglia, Italy 2006-2012. Hum Vaccin Immunother. 2015;11(1):214-9.

20. Amodio E, Tramuto F, Cracchiolo M, Sciuto V, De Donno A, Guido M, et al. The impact of ten years of infant universal varicella vaccination in Sicily, Italy (2003-2012). Hum Vaccin Immunother. 2014;11(1):236-9.

21. Marin M, Guris D, Chaves SS, Schmid S, Seward JF. Prevention of varicella: recommendations of the Advisory Committee on Immunization Practices (ACIP). MMWR Recomm Rep. 2007;56(RR-4):1-40.

22. Marin M, Zhang JX, Seward JF. Near elimination of varicella deaths in the US after implementation of the vaccination program. Pediatrics. 2011;128(2):214-20.

23. Shapiro ED, Vazquez M, Esposito D, Holabird N, Steinberg SP, Dziura J, et al. Effectiveness of 2 doses of varicella vaccine in children. J Infect Dis. 2011;203(3):312-5.

24. World Health Organization (WHO). Varicella position paper. Wkly Epidemiol Rec. 2014:89(25):265-87.

25. Klein NP, Fireman B, Yih WK, et al. Measles-mumps-rubella-varicella combination vaccine and the risk of febrile seizures. Pediatrics. 2010;126(1): e1-8. http://dx.doi.org/10.1542/peds.2010-0665.

26. Schink T, Holstiege J, Kowalzik F, et al. Risk of febrile convulsions after $M M R V$ vaccination in comparison to MMR or MMR $+V$ vaccination. Vaccine. 2014;32(6):645-50. http://dx.doi.org/10.1016/j.vaccine.2013.12.011.

27. Gavrielov-Yusim N, Hoshen M, Singer SR, et al. The weight of MMRV-related febrile convulsions among other clinical factors contributing to febrile convulsions in children. Vaccine. 2014;32(39):4954-9. http://dx.doi.org/10. 1016/j.vaccine.2014.07.024.

28. MacDonald SE, Dover DC, Simmonds KA, et al. Risk of febrile seizures after first dose of measles-mumps-rubella-varicella vaccine: a population-based cohort study. CMAJ. 2014;186(11):824-9. http://dx.doi.org/10.1503/cmaj. 140078.

29. Vitali RG. Chickenpox vaccination. Ital J Pediatr. 2014;40 Suppl 1:A3.

30. Streng A, Liese JG. Decline of varicella vaccination in German surveillance regions after recommendation of separate first-dose vaccination for varicella and measles-mumps-rubella. Vaccine. 2014;32(8):897-900.

31. Bauchau V, Van Holle L, Cohen C. Modelling hospitalisation ratios for febrile convulsions and severe varicella under combined Measles, Mumps, Rubella, and Varicella (MMRV-Priorix-Tetra ${ }^{\mathrm{TM}}$ ) compared to separate MMR $+\mathrm{V}$ vaccination. Drug Saf. 2015;38(11):1095-102.

32. Jumaan $A O, Y u O$, Jackson LA, et al. Incidence of herpes zoster, before and after varicella-vaccination-associated decreases in the incidence of varicella, 1992-2002. J Infect Dis. 2005;191(12):2002-7.

33. Kawai K, Yawn BP, Wollan P, Harpaz R. Increasing incidence of herpes zoster over a 60-year period from a population-based study. Clin Infect Dis. 2016;63(2):221-6.

34. Goldman GS. Incidence of herpes zoster among children and adolescents in a community with moderate varicella vaccination coverage. Vaccine. 2003;21(27-30):4243-9.

35. Yih WK, Brooks DR, Lett SM, et al. The incidence of varicella and herpes zoster in Massachusetts as measured by the Behavioral Risk Factor 
Surveillance System (BRFSS) during a period of increasing varicella vaccine coverage, 1998-2003. BMC Public Health. 2005;5:68.

36. Patel MS, Gebremariam A, Davis MM. Herpes zoster-related hospitalizations and expenditures before and after introduction of the varicella vaccine in the United States. Infect Control Hosp Epidemiol. 2008;29(12):1157-63.

37. Mullooly JP, Riedlinger K, Chun C, et al. Incidence of herpes zoster, 1997-2002. Epidemiol Infect. 2005;133(2):245-53.

38. Leung J, Harpaz R, Molinari NA, et al. Herpes zoster incidence among insured persons in the United States, 1993-2006: evaluation of impact of varicella vaccination. Clin Infect Dis. 2011;52(3):332-40.

39. Russell ML, Schopflocher DP, Svenson L, et al. Secular trends in the epidemiology of shingles in Alberta. Epidemiol Infect. 2007;135(6):908-13.

40. Unim B, Saulle R, Boccalini S, Taddei C, Ceccherini V, Boccia A, Bonanni P, La Torre G. Economic evaluation of Varicella vaccination: results of a systematic review. Hum Vaccin Immunother. 2013;9(9):1932-42.

Submit your next manuscript to BioMed Central and we will help you at every step:

- We accept pre-submission inquiries

- Our selector tool helps you to find the most relevant journal

- We provide round the clock customer support

- Convenient online submission

- Thorough peer review

- Inclusion in PubMed and all major indexing services

- Maximum visibility for your research

Submit your manuscript at www.biomedcentral.com/submit
Biomed Central 